\title{
A Case Report of Resection of a Mediastinal Paraganglioma: Why All the Fuss?
}

\author{
Laura Mary Staunton, M.B., B.Ch., B.A.O., Laura Casey, M.B., M.R.C.S.I., Vincent K. Young, M.D., F.R.C.S.I. (CTh), \\ Gerard J. Fitzmaurice, M.Sc., F.R.C.S.I. (CTh) \\ Department of Cardiothoracic Surgery, St. James's Hospital, Dublin, Ireland
}

\section{ARTICLE INFO}

Received September 29, 2021

Revised November 11, 202

Accepted November 17, 2021

Corresponding author

Laura Mary Staunton

Tel 353-857573827

Fax 353-1-410-3700

E-mail laurastaunton@rcsi.ie

ORCID

https://orcid.org/0000-0002-9006-3333
Mediastinal paragangliomas are rare tumors that have only been reported in individual cases or limited case series. Surgical resection of these tumors can be challenging, as they are highly vascular and intimately related to the great vessels. Surgery is usually performed via median sternotomy with or without cardiopulmonary bypass. We present the case of a mediastinal paraganglioma that was resected via a left-sided posterolateral thoracotomy. Histopathology revealed a completely resected 38-mm paraganglioma with a positive station 5 lymph node, indicative of locally aggressive disease. Hereditary paragangliomas are associated with malignant transformation; therefore, genetic testing is important. These tumors do not respond well to chemoradiotherapy, and consequently lifelong surveillance for early detection of recurrence is recommended.

Keywords: Lung neoplasm, Mediastinal paraganglioma, Posterolateral thoracotomy, SDHB mutation, Case report

\section{Case report}

Mediastinal paragangliomas originate from the chromaffin cells of the extra-adrenal sympathetic ganglia, which are located around the great vessels [1-3]. These rare tumors represent only $0.3 \%$ of mediastinal tumors and $1 \%$ $2 \%$ of all paragangliomas $[1,4,5]$. They can be located in the posterior mediastinum (known as aorticosympathetic) or the anterior and middle mediastinum (aorticopulmonary) $[1,3,5]$. Surgical resection can be extremely challenging, as these tumors are highly vascular and have an intimate relationship with the great vessels, heart, trachea, and esophagus $[1,3,5]$. We present a case of a mediastinal paraganglioma that was incidentally found on screening thoracic computed tomography (CT) and was safely resected via a left-sided posterolateral thoracotomy.

A 53-year-old woman was incidentally found to have a mediastinal paraganglioma during a screening thoracic CT for investigation of a chronic cough. She reported a previous history of hyperthyroidism and was a non-smoker. Thoracic $\mathrm{CT}$ demonstrated a $3.5 \times 4.2 \mathrm{~cm}$ mass that was contiguous with the aortic arch and left pulmonary artery (LPA)
(Fig. 1A). Positron emission tomography (PET)-CT showed a fluorodeoxyglucose-avid $4.5 \times 4.0 \mathrm{~cm}$ left-sided middle mediastinal mass (Fig. 1B). CT-guided biopsy of this mass favored a paraganglioma, as nested cells with pale eosinophilic granular cytoplasm were revealed. Immunohistochemistry demonstrated cells positive for chromogranin and synaptophysin; S100 stained some cells. Biochemical tests were unremarkable. The cellular appearance in com-
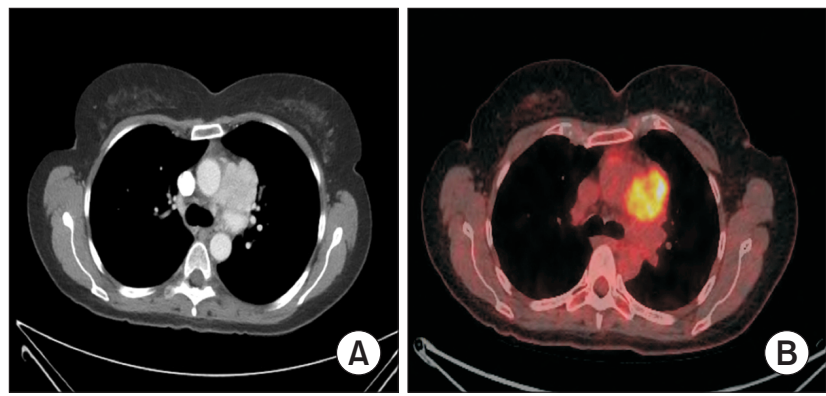

Fig. 1. Axial slices of (A) contrast-enhanced thoracic computed tomography $(\mathrm{CT})$ and (B) positron emission tomography-CT showing a left middle mediastinal mass adherent to the aortic arch and left pulmonary artery. 

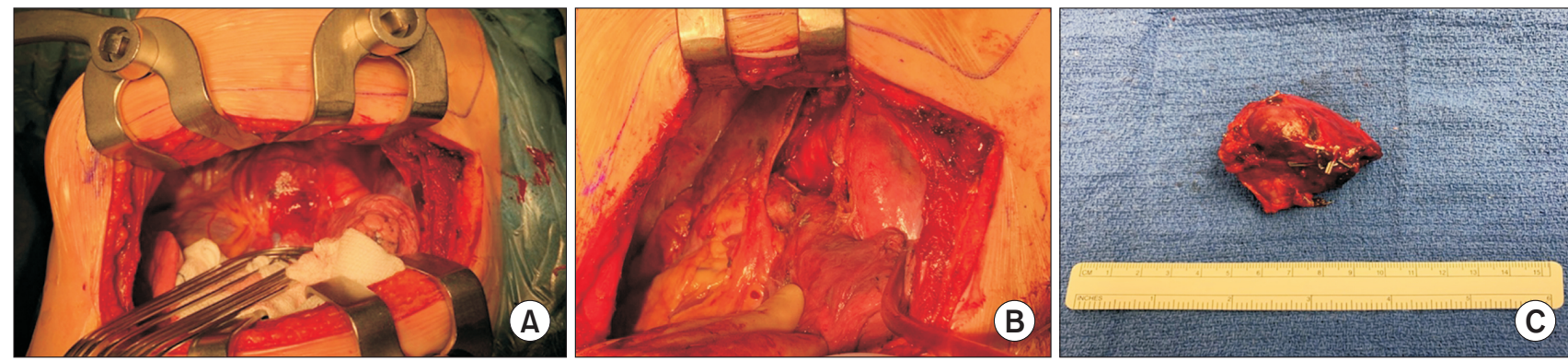

Fig. 2. (A) The initial appearance prior to resection demonstrating a mass adherent to the aortic arch with the phrenic nerve visible anteriorly, and (B) post-resection findings with no evidence of residual tumor between the aortic arch and left pulmonary artery. (C) The completely resected 38-mm paraganglioma.

bination with the immunohistochemistry, and in conjunction with the radiological findings, led to a diagnosis of primary mediastinal paraganglioma. Preoperative planning at a multidisciplinary team meeting led to the decision to excise the paraganglioma via a left-sided posterolateral thoracotomy with the possibility of a staged median sternotomy rather than a hemi-clamshell incision. The left recurrent laryngeal nerve (RLN) was expected to be sacrificed. Cardiopulmonary bypass would remain on standby.

During surgery, a large extensively vascular mass abutting the aortic arch and proximal LPA, with extension into the aorto-pulmonary window, was resected via a left postero-lateral thoracotomy (Fig. 2A, B). The mass was dissected free from the phrenic and vagus nerves, which were both preserved. The RLN was sacrificed. The pericardium was opened over the pulmonary artery trunk and dissection was performed from the aortic arch. The tumor was then dissected from the LPA. A station 5 lymph node was completely excised. Final histopathology revealed a $38-\mathrm{mm}$ paraganglioma with an $\mathrm{R} 0$ resection (Fig. 2C) and direct invasion of an adjacent lymph node and the separately submitted station 5 lymph node; these findings were indicative of aggressive local disease.

Postoperatively the patient experienced hoarseness. On the second postoperative day, she underwent left vocal cord medialization due to the expected left vocal cord palsy. The chest drain was removed on postoperative day 1 and she was discharged home on postoperative day 6 in good health.

The patient provided written informed consent for publication of her clinical details and images.

\section{Discussion}

Only case reports or small case series have been reported for mediastinal paragangliomas $[1,3,6]$. The gold-standard treatment remains complete surgical resection, with a longterm survival rate of approximately $84 \%[1,4,7-9]$. The preoperative diagnosis of mediastinal paragangliomas is of the utmost importance to allow appropriate treatment planning. Imaging modalities, in particular CT and PET-CT, showed a middle mediastinal mass. Tissue diagnosis was obtained following a CT-guided biopsy with histopathological findings consistent with a mediastinal paraganglioma. The surgery itself posed the technical challenge of excising the tumor, as this highly vascular mass was abutting the aortic arch and proximal LPA and extended into the aorto-pulmonary window. Surgery is usually performed via median sternotomy with or without cardiopulmonary bypass for anterior and middle mediastinal tumors or a posterolateral thoracotomy for posterior tumors $[1,4,6,7]$. A single case report described resection of a mediastinal paraganglioma via an anterior thoracotomy, as well as by video-assisted thoracoscopic surgery in smaller tumors $[9,10]$. This case demonstrates that resection of a middle mediastinal tumor can be safely undertaken via a left-sided posterolateral thoracotomy in appropriately selected cases with comprehensive preoperative planning.

In total, $15 \%-35 \%$ of paragangliomas have been shown to undergo malignant transformation and recurrence occurs in approximately $20 \%$ of cases [5]. As histological findings cannot determine malignancy, metastasis by definition indicates tumor malignancy, as in this case [5]. In general, lifelong surveillance is recommended and chemoradiotherapy regimens are not standardized and largely ineffective [5]. Even so, paragangliomas are not aggressive in nature (i.e., not truly benign or malignant) and the overall survival for metastatic tumors is 24.6 years [5]. Twenty-five percent of paragangliomas are familial, and hereditary paragangliomas commonly show mutations of the succinate dehydrogenase subunit $(S D H B)[4,6]$. SDHB mutation increases the likelihood of malignant transformation [6]; 
therefore, it is important to refer patients for genetic testing.

In conclusion, safe resection of middle mediastinal paraganglioma can be undertaken via a posterolateral thoracotomy, without cardiopulmonary bypass, in select cases. Metastasis can occur, as in this case; however, these tumors are not malignantly aggressive. Due to their poor response to chemoradiotherapy, lifelong surveillance for the early detection of recurrence is recommended.

\section{Conflict of interest}

No potential conflict of interest relevant to this article was reported.

\section{ORCID}

Laura Mary Staunton: https://orcid.org/0000-0002-9006-3333 Laura Casey: https://orcid.org/0000-0002-0745-3008

Vincent K. Young: https://orcid.org/0000-0003-2279-2697

Gerard J. Fitzmaurice: https://orcid.org/0000-0002-0768-6709

\section{References}

1. Shah MUA, Al-Saadi N, Leatherby R, Xylas D, Shah S. Aortopulmonary paraganglioma masquerading as an anterior mediastinal mass: a fine line between confusion and chaos! J Card Surg 2020;35: 2044-6.

2. Wald O, Shapira OM, Murar A, Izhar U. Paraganglioma of the mediastinum: challenges in diagnosis and surgical management. J Cardiothorac Surg 2010;5:19.

3. Nguyen DM, Gonzalez JN, Villamizar NR. Surgical management of mediastinal paraganglioma: all hands on deck! J Card Surg 2020;35: 2047-9.

4. Buchanan SN, Radecki KM, Chambers LW. Mediastinal paraganglioma. Ann Thorac Surg 2017;103:e413-4.

5. Rahi MS, Gunasekaran K, Amoah K, Rudolph D. Paraganglioma of the middle mediastinum. Respir Med Case Rep 2020;31:101211.

6. Brown ML, Zayas GE, Abel MD, Young WF Jr, Schaff HV. Mediastinal paragangliomas: the Mayo Clinic experience. Ann Thorac Surg 2008;86:946-51.

7. Chang SH, Yapar I, Kozower BD. Aorticopulmonary paraganglioma with symptomatic postoperative bradycardia. Ann Thorac Surg 2020; 109:e367-9.

8. Hato T, Kaseda K, Harada M, Horio H. Aorticopulmonary paraganglioma. Gen Thorac Cardiovasc Surg 2011;59:812-4.

9. Shakir M, Blossom G, Lippert J. Anterior mediastinal paraganglioma: a case for preoperative embolization. World J Surg Oncol 2012; 10:134.

10. Kim D, Kim SW, Hong JM. Mediastinal paraganglioma: complete resection using video-assisted thoracoscopic surgery. Korean J Thorac Cardiovasc Surg 2014;47:197-9. 